Science

\title{
AMENDED PARTICLE SWARM OPTIMIZATION ALGORITHM FOR REAL POWER LOSS REDUCTION AND STATIC VOLTAGE STABILITY MARGIN INDEX ENHANCEMENT
}

\author{
Dr.K.Lenin *1 \\ ${ }^{* 1}$ Professor, Department of EEE Prasad V.Potluri Siddhartha Institute of Technology, Kanuru, \\ Vijayawada, Andhra Pradesh -520007, India
}

\begin{abstract}
In this paper, Amended Particle Swarm Optimization Algorithm (APSOA) is proposed with the combination of Particle Swarm Optimization (PSO) and Gravitational Search Algorithm (GSA) for solving the optimal reactive power dispatch Problem. PSO is one of the most widely used evolutionary algorithms in hybrid methods due to its simplicity, convergence speed, an ability of searching Global optimum. GSA has many advantages such as, adaptive learning rate, memoryless algorithm and, good and fast convergence. Proposed hybridized algorithm is aimed at reduce the probability of trapping in local optimum. In order to assess the efficiency of proposed algorithm, it has been tested on Standard IEEE 30 system and compared to other standard algorithms. The simulation results demonstrate worthy performance of the Amended Particle Swarm Optimization Algorithm (APSOA) in solving optimal reactive power dispatch problem.
\end{abstract}

Keywords: Gravitational Search Algorithm (GSA); Particle Swarm Optimization (PSO); Optimal Reactive Power Dispatch.

Cite This Article: Dr.K. Lenin. (2018). "AMENDED PARTICLE SWARM OPTIMIZATION ALGORITHM FOR REAL POWER LOSS REDUCTION AND STATIC VOLTAGE STABILITY MARGIN INDEX ENHANCEMENT." International Journal of Research Granthaalayah, 6(2), 146-156. https://doi.org/10.5281/zenodo.1189217.

\section{Introduction}

In recent years the optimal reactive power dispatch (ORPD) problem has received great attention as a result of the improvement on economy and security of power system operation. Gradient method [1, 2] Newton method [3] and linear programming [4-6] like various mathematical techniques have been adopted to solve the optimal reactive power dispatch problem. But they have difficulty in handling inequality constraints. Many Evolutionary algorithms such as have been proposed to solve the reactive power dispatch problem [7-10]. In this paper, Amended Particle Swarm Optimization Algorithm (APSOA) is proposed with the combination of Particle Swarm Optimization (PSO) and Gravitational Search Algorithm (GSA) for solving the optimal reactive power dispatch Problem. Merging the optimization algorithms is a way to balance the 
overall exploration and Exploitation ability. PSO is one of the most widely used evolutionary algorithms in hybrid methods due to its simplicity, convergence speed, an ability of searching Global optimum. GSA has many advantages [11] such as, adaptive learning rate, memory-less algorithm and, good and fast convergence. There are some studies in the literature [12-19] which have been done to synthesize PSO with other algorithms. These hybrid algorithms are aimed at reducing the probability of trapping in local optimum. PSO is one of the most widely used evolutionary algorithms in hybrid methods due to its simplicity, convergence speed, an ability of searching Global optimum. GSA has many advantages such as, adaptive learning rate, memoryless algorithm and, good and fast convergence. This hybridized algorithm is aimed at reduce the probability of trapping in local optimum. In order to assess the efficiency of proposed algorithm, it has been tested on Standard IEEE 30 system and compared to other standard algorithms. The simulation results demonstrate worthy performance of the Amended Particle Swarm Optimization Algorithm (APSOA) in solving optimal reactive power dispatch problem.

\section{Voltage Stability Evaluation}

\subsection{Modal Analysis for Voltage Stability Evaluation}

Power flow equations of the steady state system is given by,

$\left[\begin{array}{l}\Delta \mathrm{P} \\ \Delta \mathrm{Q}\end{array}\right]=\left[\begin{array}{cc}\mathrm{J}_{\mathrm{p} \theta} & \mathrm{J}_{\mathrm{pv}} \\ \mathrm{J}_{\mathrm{q} \theta} & \mathrm{J}_{\mathrm{QV}}\end{array}\right]\left[\begin{array}{l}\Delta \theta \\ \Delta V\end{array}\right]$

Where

$\Delta \mathrm{P}=$ bus real power change incrementally.

$\Delta \mathrm{Q}=$ bus reactive Power injection change incrementally.

$\Delta \theta=$ bus voltage angle change incrementally.

$\Delta \mathrm{V}=$ bus voltage Magnitude change incrementally.

$\mathrm{Jp} \theta$, JPV , JQ $\theta$, JQV are sub-matrixes of the System voltage stability in jacobian matrix and both $\mathrm{P}$ and $\mathrm{Q}$ get affected by this.

Presume $\Delta \mathrm{P}=0$, then equation (1) can be written as, $\Delta \mathrm{Q}=\left[\mathrm{J}_{\mathrm{QV}}-\mathrm{J}_{\mathrm{Q} \theta} \mathrm{J}_{\mathrm{P}^{-1}} \mathrm{~J}_{\mathrm{PV}}\right] \Delta \mathrm{V}=\mathrm{J}_{\mathrm{R}} \Delta \mathrm{V}$

$\Delta \mathrm{V}=\mathrm{J}^{-1}-\Delta \mathrm{Q}$

Where

$\mathrm{J}_{\mathrm{R}}=\left(\mathrm{J}_{\mathrm{QV}}-\mathrm{J}_{\mathrm{Q} \theta} \mathrm{J}_{\mathrm{P} \theta^{-1}} \mathrm{JPV}\right)$

$\mathrm{J}_{\mathrm{R}}$ denote the reduced Jacobian matrix of the system.

\subsection{Modes of Voltage Instability}

Voltage Stability characteristics of the system have been identified through computation of the Eigen values and Eigen vectors.

$\mathrm{J}_{\mathrm{R}}=\xi \wedge \eta$ 
Where,

$\xi$ denote the right eigenvector matrix of JR, $\eta$ denote the left eigenvector matrix of JR, $\wedge$ denote the diagonal eigenvalue matrix of JR.

$\mathrm{J}_{\mathrm{R}^{-1}}=\xi \wedge^{-1} \eta$

From the equations (5) and (6),

$\Delta \mathrm{V}=\xi \wedge^{-1} \eta \Delta \mathrm{Q}$

Or

$\Delta \mathrm{V}=\sum_{\mathrm{I}} \frac{\xi_{\mathrm{i}} \eta_{\mathrm{i}}}{\lambda_{\mathrm{i}}} \Delta \mathrm{Q}$

$\xi i$ denote the ith column right eigenvector $\& \eta$ is the ith row left eigenvector of JR.

$\lambda \mathrm{i}$ indicate the ith Eigen value of JR.

reactive power variation of the ith modal is given by,

$\Delta \mathrm{Q}_{\mathrm{mi}}=\mathrm{K}_{\mathrm{i}} \xi_{\mathrm{i}}$

where,

$\mathrm{K}_{\mathrm{i}}=\sum_{\mathrm{j}} \xi_{\mathrm{ij}}{ }^{2}-1$

Where $\xi \mathrm{ji}$ is the jth element of $\xi \mathrm{i}$

ith modal voltage variation is mathematically given by,

$\Delta \mathrm{V}_{\mathrm{mi}}=\left[1 / \lambda_{\mathrm{i}}\right] \Delta \mathrm{Q}_{\mathrm{mi}}$

When the value of $|\lambda \mathrm{i}|=0$ then the ith modal voltage will get collapsed.

In equation (8), when $\Delta \mathrm{Q}=\mathrm{ek}$ is assumed, then ek has all its elements zero except the kth one being 1 . Then $\Delta \mathrm{V}$ can be formulated as follows,

$$
\Delta \mathrm{V}=\sum_{\mathrm{i}} \frac{\eta_{1 \mathrm{k} \xi_{1}}}{\lambda_{1}}
$$

$\eta_{1 \mathrm{k}}$ is $\mathrm{k}$ th element of $\eta_{1}$

At bus $\mathrm{k} V-\mathrm{Q}$ sensitivity is given by,

$\frac{\partial \mathrm{V}_{\mathrm{K}}}{\partial \mathrm{Q}_{\mathrm{K}}}=\sum_{\mathrm{i}} \frac{\eta_{1 \mathrm{k}} \xi_{1}}{\lambda_{1}}=\sum_{\mathrm{i}} \frac{\mathrm{P}_{\mathrm{ki}}}{\lambda_{1}}$ 


\section{Problem Formulation}

To minimize the real power loss and also to maximize the static voltage stability margin (SVSM) is the key objectives of the reactive power dispatch problem.

\subsection{Minimization of Real Power Loss}

Real power loss (Ploss) minimization in transmission lines is mathematically given as,

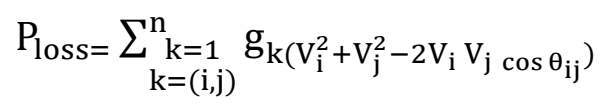

Where $\mathrm{n}$ is the number of transmission lines, $\mathrm{gk}$ is the conductance of branch $\mathrm{k}, \mathrm{Vi}$ and $\mathrm{Vj}$ are voltage magnitude at bus $i$ and bus $j$, and $\theta \mathrm{ij}$ is the voltage angle difference between bus $\mathrm{i}$ and bus j.

\subsection{Minimization of Voltage Deviation}

At load buses minimization of the voltage deviation magnitudes (VD) is stated as follows,

Minimize $V D=\sum_{\mathrm{k}=1}^{\mathrm{nl}}\left|\mathrm{V}_{\mathrm{k}}-1.0\right|$

Where $\mathrm{nl}$ is the number of load busses and $\mathrm{Vk}$ is the voltage magnitude at bus $\mathrm{k}$.

\subsection{System Constraints}

These are the following constraints subjected to objective function as given below, Load flow equality constraints:

$P_{G i}-P_{D i}-V_{i} \sum_{j=1}^{n b} v_{j}\left[\begin{array}{cc}G_{i j} & \cos \theta_{i j} \\ +B_{i j} & \sin \theta_{i j}\end{array}\right]=0, i=1,2 \ldots, n b$

$Q_{G i}-Q_{D i}-V_{i} \sum_{j=1}^{n b} v_{j}\left[\begin{array}{cc}G_{i j} & \sin \theta_{i j} \\ +B_{i j} & \cos \theta_{i j}\end{array}\right]=0, i=1,2 \ldots, n b$

where, $\mathrm{nb}$ is the number of buses, $\mathrm{P}_{\mathrm{G}}$ and $\mathrm{Q}_{\mathrm{G}}$ are the real and reactive power of the generator, $\mathrm{P}_{\mathrm{D}}$ and $Q_{D}$ are the real and reactive load of the generator, and $G_{i j}$ and $B_{i j}$ are the mutual conductance and susceptance between bus $i$ and bus $j$.

Generator bus voltage $\left(\mathrm{V}_{\mathrm{Gi}}\right)$ inequality constraint:

$\mathrm{V}_{\mathrm{Gi}}^{\min } \leq \mathrm{V}_{\mathrm{Gi}} \leq \mathrm{V}_{\mathrm{Gi}}^{\max }, \mathrm{i} \in \mathrm{ng}$

Load bus voltage $\left(\mathrm{V}_{\mathrm{Li}}\right)$ inequality constraint: 
$\mathrm{V}_{\mathrm{Li}}^{\min } \leq \mathrm{V}_{\mathrm{Li}} \leq \mathrm{V}_{\mathrm{Li}}^{\max }, \mathrm{i} \in \mathrm{nl}$

Switchable reactive power compensations $\left(\mathrm{Q}_{\mathrm{Ci}}\right)$ inequality constraint:

$\mathrm{Q}_{\mathrm{Ci}}^{\min } \leq \mathrm{Q}_{\mathrm{Ci}} \leq \mathrm{Q}_{\mathrm{Ci}}^{\max }, \mathrm{i} \in \mathrm{nc}$

Reactive power generation $\left(\mathrm{Q}_{\mathrm{Gi}}\right)$ inequality constraint:

$\mathrm{Q}_{\mathrm{Gi}}^{\min } \leq \mathrm{Q}_{\mathrm{Gi}} \leq \mathrm{Q}_{\mathrm{Gi}}^{\mathrm{max}}, \mathrm{i} \in \mathrm{ng}$

Transformers tap setting $\left(\mathrm{T}_{\mathrm{i}}\right)$ inequality constraint:

$\mathrm{T}_{\mathrm{i}}^{\min } \leq \mathrm{T}_{\mathrm{i}} \leq \mathrm{T}_{\mathrm{i}}^{\max }, \mathrm{i} \in \mathrm{nt}$

Transmission line flow $\left(\mathrm{S}_{\mathrm{Li}}\right)$ inequality constraint:

$\mathrm{S}_{\mathrm{Li}}^{\min } \leq \mathrm{S}_{\mathrm{Li}}^{\max }, \mathrm{i} \in \mathrm{nl}$

Where, nc, ng and nt are numbers of the switchable reactive power sources, generators and transformers.

\section{Particle Swarm Optimization}

Particle Swarm Optimization (PSO) is an evolutionary computation technique which is inspired from social behavior of bird flocking. It uses a number of particles (candidate solutions) which fly around in the search space to find best solution. Meanwhile, they all look at the best particle (best solution) in their paths. In other words, particles consider their own best solutions as well as the best solution has found so far. Each particle in PSO should consider the current position, the current velocity, the distance to pbest, and the distance to gbest to modify its position. PSO was mathematically modeled as follow:

$$
\begin{aligned}
v_{i}^{t+1} & =w v_{i}^{t}+c_{1} \times \text { rand } \times\left(\text { pbest }_{i}-x_{i}^{t}\right)+c_{2} \times \text { rand } \times\left(\text { gbest }-x_{i}^{t}\right) \\
x_{i}^{t+1} & =x_{i}^{t}+v_{i}^{t+1}
\end{aligned}
$$

The PSO starts with randomly placing the particles in a problem space. In each iteration, the velocities of particles are calculated using equation (24). After defining the velocities, the position of masses can be calculated by equation (25). The process of changing particles' position will continue until meeting an end criterion.

\section{Gravitational Search Algorithm}

Gravitational Search Algorithm (GSA) is a novel heuristic optimization method which is inspired from is the Newton's theory that states: Every particle in the universe attracts every other particle with a force that is directly proportional to the product of their masses and inversely proportional to the square of the distance between them. GSA can be considered as a collection of agents 
(candidate solutions) whose have masses proportional to their value of fitness function. During generations, all masses attract each other by the gravity forces between them. A heavier mass has the bigger attraction force. Therefore, the heavier masses which are probably close to the global Optimum attract the other masses proportional to their Distances. The GSA was mathematically modeled as follow. Suppose a system with $\mathrm{N}$ agents. The algorithm starts with randomly placing all agents in search space. During all epochs, the gravitational forces from agent $j$ on agent $i$ at a Specific time $t$ is defined as follows;

$F_{i j}^{d}(t)=G(t) \frac{M_{p i}(t) \times M_{a j}(t)}{R_{i j}(t)+\epsilon}\left(x_{j}^{d}(t)-x_{i}^{d}(t)\right)$

The $\mathrm{G}(\mathrm{t})$ is calculated as $(27)$ :

$$
G(t)=G_{0} \times \exp \left(-a \times \frac{\text { iter }}{\text { maxiter }}\right)
$$

In a problem space with the dimension $d$, the total force that acts on agent $i$ is calculated as the following equation:

$$
F_{i}^{d}(t)=\sum_{i=1, j \neq i}^{N} \operatorname{rand}_{j} F_{i j}^{d}(t)
$$

According to the law of motion, the acceleration of an agent is proportional to the result force and inverse of its mass, so the acceleration of all agents should be calculated as follow:

$a c_{i}^{d}(t)=\frac{F_{i}^{d}(t)}{M_{i i}(t)}$

The velocity and position of agents are calculated as follows:

$$
\begin{gathered}
\operatorname{vel}_{i}^{d}(t+1)=\operatorname{rand}_{i} \times \operatorname{vel}_{i}^{d}(t)+a c_{i}^{d}(t) \\
x_{i}^{d}(t+1)=x_{i}^{d}(t)+\operatorname{vel}_{i}^{d}(t+1)
\end{gathered}
$$

In GSA, at first all masses are initialized with random Values. Each mass is a candidate solution. After Initialization, velocities for all masses are defined using (30). Meanwhile the gravitational constant, total forces, and accelerations are calculated as (27), (28), and (29) respectively. The positions of masses are calculated using (31). Finally GSA will be stopped by meeting an end criterion.

\section{Amended Particle Swarm Optimization Algorithm (APSOA)}

Two algorithms can be hybridized in high-level or low-level with relay or co evolutionary method as homogeneous or heterogeneous. In this paper, we hybridize PSO with GSA using low-level co evolutionary heterogeneous hybrid. The hybrid is low-level because we combine the functionality of both algorithms. It is co-evolutionary because we do not use both algorithm one after another. In other words, they run in parallel. It is heterogeneous because there are two different algorithms that are involved to produce final results. The basic idea of proposed 
algorithm is to combine the ability of social thinking (gbest) in PSO with the local search Capability of GSA. In order to combine these algorithms, (32) is proposed as follow:

$$
V_{i}(t+1)=w \times V_{i}(t)+C_{1}^{\prime} \times \text { rand } \times a c_{i}(t)+C_{2}^{\prime} \times \operatorname{rand} \times\left(g b e s t-X_{i}(t)\right)
$$

In each iteration, the positions of particles are updated as follow:

$$
X_{i}(t+1)=X_{i}(t)+V_{i}(t+1)
$$

In the proposed algorithm, at first, all agents are randomly initialized. Each agent is considered as a candidate solution. After initialization, Gravitational force, gravitational constant, and resultant forces among agents are calculated using (26), (27), and (28) respectively. After that, the accelerations of particles are defined as (29). In each iteration, the best solution so far should be updated. After calculating the accelerations and with updating the best solution so far, the velocities of all agents can be calculated using (32). Finally, the positions of agents are defined as (33). The process of updating velocities and positions will be stopped by meeting an end criterion. The steps of hybridized algorithm are represented in fig.1.

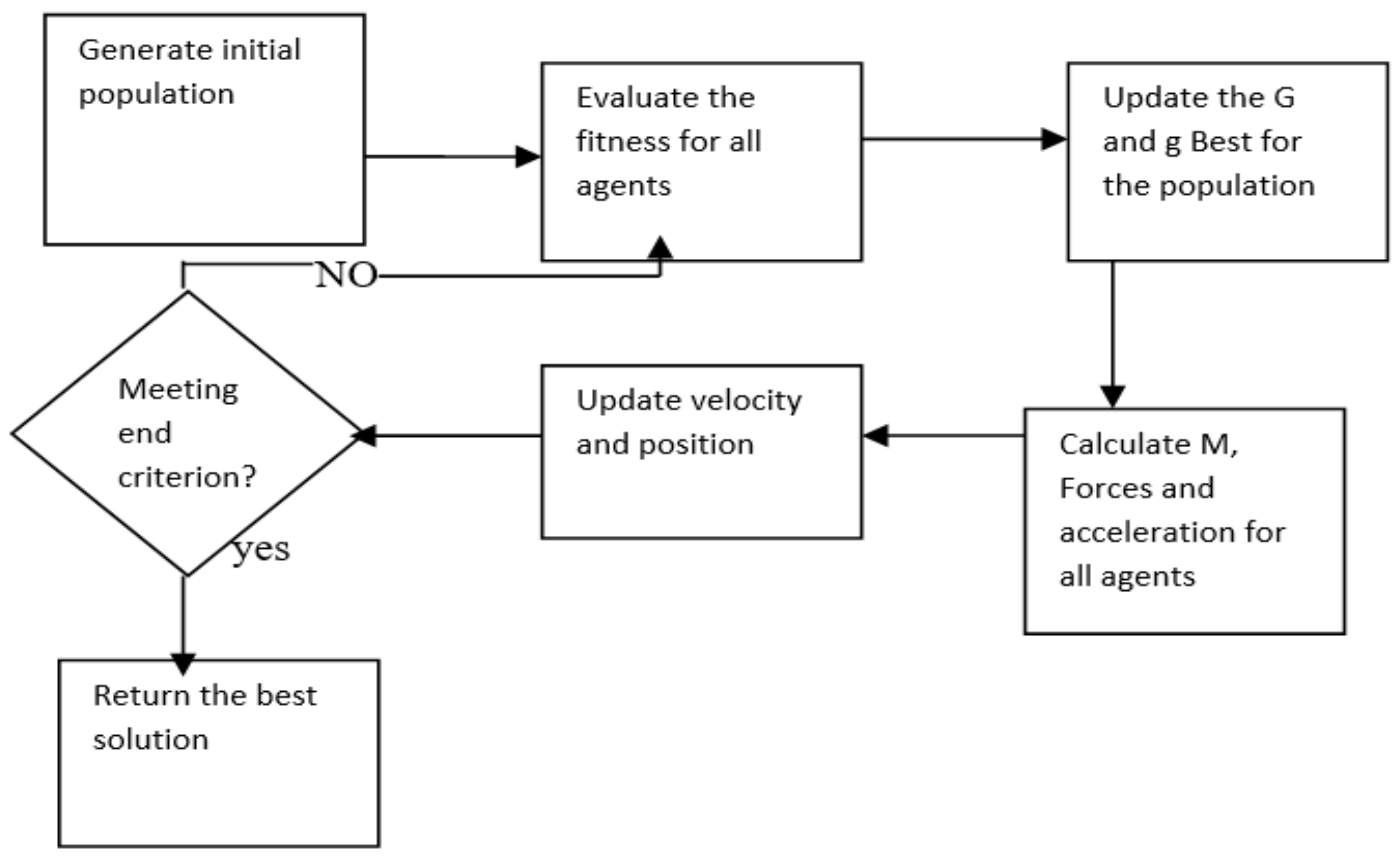

Figure 1: Steps of Hybridized PSO \& GSA algorithm

In Amended Particle Swarm Optimization Algorithm (APSOA), the quality of solutions (fitness) is considered in the updating procedure. The agents near good solutions try to attract the other agents which are exploring the search space. When all agents are near a good solution, they move very slowly. In this case, the gBest help them to exploit the global best. Amended Particle Swarm Optimization Algorithm (APSOA) use a memory (gBest) to save the best solution has found so far, so it is accessible anytime. 


\section{Simulation Results}

The efficiency of the proposed Amended Particle Swarm Optimization Algorithm (APSOA) is demonstrated by testing it on standard IEEE-30 bus system. 6 generator buses, 24 load buses and 41 transmission lines of which four branches are (6-9), (6-10), (4-12) and (28-27) - are with the tap setting transformers in standard IEEE-30 bus system. Lower voltage magnitude limits at all buses are 0.95 p.u. and the upper limits are 1.1 for all the PV buses, for PQ buses \& reference bus it is 1.05 p.u.. Comparisons of results are shown in Table 5. In Table 1 optimal values of the control variables are given.

Table 1: Results of APSOA - ORPD optimal control variables

\begin{tabular}{|l|l|}
\hline Control variables & Values of Variable setting \\
\hline V1 & 1.0402 \\
V2 & 1.0411 \\
V5 & 1.0420 \\
V8 & 1.0301 \\
V11 & 1.0020 \\
V13 & 1.0301 \\
T11 & 1.000 \\
T12 & 1.000 \\
T15 & 1.010 \\
T36 & 1.010 \\
Qc10 & 2 \\
Qc12 & 3 \\
Qc15 & 2 \\
Qc17 & 0 \\
Qc20 & 2 \\
Qc23 & 2 \\
Qc24 & 3 \\
Qc29 & 2 \\
Real power loss & 4.2006 \\
SVSM & 0.2486 \\
\hline
\end{tabular}

Table 2 indicates the optimal values of the control variables \& there is no limit violations in state variables. Mainly static voltage stability margin (SVSM) has increased from 0.2486 to 0.2498 . contingency analysis was conducted using the control variable setting obtained in case 1 and case 2 to determine the voltage security of the system. In Table 3 the Eigen values equivalents to the four critical contingencies are given. Result reveal about the Eigen value has been improved considerably for all contingencies in the second case. 
Table 2: Results of APSOA - Voltage Stability Control Reactive Power Dispatch Optimal Control Variables

\begin{tabular}{|l|l|}
\hline Control Variables & Values of Variable Setting \\
\hline V1 & 1.0450 \\
V2 & 1.0471 \\
V5 & 1.0480 \\
V8 & 1.0300 \\
V11 & 1.0032 \\
V13 & 1.0324 \\
T11 & 0.090 \\
T12 & 0.090 \\
T15 & 0.090 \\
T36 & 0.090 \\
Qc10 & 3 \\
Qc12 & 2 \\
Qc15 & 2 \\
Qc17 & 3 \\
Qc20 & 0 \\
Qc23 & 2 \\
Qc24 & 2 \\
Qc29 & 3 \\
Real power loss & 4.9892 \\
SVSM & 0.2498 \\
\hline
\end{tabular}

Table 3: Voltage Stability under Contingency State

\begin{tabular}{|l|l|l|l|}
\hline Sl.No & Contingency & $\begin{array}{l}\text { Optimal Reactive Power } \\
\text { Dispatch Setting }\end{array}$ & $\begin{array}{l}\text { Voltage Stability Control Reactive } \\
\text { Power Dispatch Setting }\end{array}$ \\
\hline 1 & $28-27$ & 0.1419 & 0.1424 \\
\hline 2 & $4-12$ & 0.1642 & 0.1651 \\
\hline 3 & $1-3$ & 0.1761 & 0.1764 \\
\hline 4 & $2-4$ & 0.2022 & 0.2052 \\
\hline
\end{tabular}

Table 4: Limit Violation Checking Of State Variables

\begin{tabular}{|l|l|l|l|l|}
\hline \multirow{2}{*}{$\begin{array}{l}\text { State } \\
\text { variables }\end{array}$} & \multicolumn{2}{|c|}{ Limits } & $\begin{array}{l}\text { Optimal Reactive Power } \\
\text { Dispatch Setting }\end{array}$ & $\begin{array}{l}\text { Voltage Stability Control } \\
\text { Reactive Power Dispatch Setting }\end{array}$ \\
\hline Q1 & -20 & 152 & 1.3422 & -1.3269 \\
\hline Q2 & -20 & 61 & 8.9900 & 9.8232 \\
\hline Q5 & -15 & 49.92 & 25.920 & 26.001 \\
\hline Q8 & -10 & 63.52 & 38.8200 & 40.802 \\
\hline Q11 & -15 & 42 & 2.9300 & 5.002 \\
\hline Q13 & -15 & 48 & 8.1025 & 6.033 \\
\hline V3 & 0.95 & 1.05 & 1.0372 & 1.0392 \\
\hline V4 & 0.95 & 1.05 & 1.0307 & 1.0328 \\
\hline V6 & 0.95 & 1.05 & 1.0282 & 1.0298 \\
\hline V7 & 0.95 & 1.05 & 1.0101 & 1.0152 \\
\hline V9 & 0.95 & 1.05 & 1.0462 & 1.0412 \\
\hline
\end{tabular}




\begin{tabular}{|l|l|l|l|l|}
\hline V10 & 0.95 & 1.05 & 1.0482 & 1.0498 \\
\hline V12 & 0.95 & 1.05 & 1.0400 & 1.0466 \\
\hline V14 & 0.95 & 1.05 & 1.0474 & 1.0443 \\
\hline V15 & 0.95 & 1.05 & 1.0457 & 1.0413 \\
\hline V16 & 0.95 & 1.05 & 1.0426 & 1.0405 \\
\hline V17 & 0.95 & 1.05 & 1.0382 & 1.0396 \\
\hline V18 & 0.95 & 1.05 & 1.0392 & 1.0400 \\
\hline V19 & 0.95 & 1.05 & 1.0381 & 1.0394 \\
\hline V20 & 0.95 & 1.05 & 1.0112 & 1.0194 \\
\hline V21 & 0.95 & 1.05 & 1.0435 & 1.0243 \\
\hline V22 & 0.95 & 1.05 & 1.0448 & 1.0396 \\
\hline V23 & 0.95 & 1.05 & 1.0472 & 1.0372 \\
\hline V24 & 0.95 & 1.05 & 1.0484 & 1.0372 \\
\hline V25 & 0.95 & 1.05 & 1.0142 & 1.0192 \\
\hline V26 & 0.95 & 1.05 & 1.0494 & 1.0422 \\
\hline V27 & 0.95 & 1.05 & 1.0472 & 1.0452 \\
\hline V28 & 0.95 & 1.05 & 1.0243 & 1.0283 \\
\hline V29 & 0.95 & 1.05 & 1.0439 & 1.0419 \\
\hline V30 & 0.95 & 1.05 & 1.0418 & 1.0397 \\
\hline
\end{tabular}

Table 5: Comparison of Real Power Loss

\begin{tabular}{|l|l|}
\hline Method & Minimum loss \\
\hline Evolutionary programming [20] & 5.0159 \\
\hline Genetic algorithm [21] & 4.665 \\
\hline Real coded GA with Lindex as SVSM[22] & 4.568 \\
\hline Real coded genetic algorithm [23] & 4.5015 \\
\hline Proposed APSOA method & 4.2006 \\
\hline
\end{tabular}

\section{Conclusion}

In this paper, Amended Particle Swarm Optimization Algorithm (APSOA) has been successfully solved the optimal reactive power dispatch problem. Proposed hybridized algorithm is aimed at reduce the probability of trapping in local optimum. In order to assess the efficiency of proposed algorithm, it has been tested on Standard IEEE 30 system and compared to other standard algorithms. The simulation results demonstrate worthy performance of the Amended Particle Swarm Optimization Algorithm (APSOA) in solving optimal reactive power dispatch problem. Real power loss has been reduced \& static voltage stability margin index has been enhanced.

\section{References}

[1] M. A. Abido, J. M. Bakhashwain, "A novel multi objective evolutionary algorithm for optimal reactive power dispatch problem," in proc. Electronics, Circuits and Systems conf., vol. 3, pp. 1054-1057, 2003.

[2] W. N. W. Abdullah, H. Saibon, A. A. M. Zain, K. L. Lo, "Genetic Algorithm for Optimal Reactive Power Dispatch," in proc. Energy Management and Power Delivery conf., vol. 1, pp. 160-164, 1998. 
[3] K. Y. Lee, Y. M. Park, J. L. Ortiz, "Fuel-cost minimisation for both real and reactive-power dispatches," in proc. Generation, Transmission and Distribution conf., vol. 131, pp. 85-93, 1984.

[4] S. Granville, "Optimal Reactive Dispatch Trough Interior Point Methods," IEEE Trans. on Power Systems, vol. 9, pp. 136-146, 1994.

[5] N. I. Deeb, S. M. Shahidehpour, "An Efficient Technique for Reactive Power Dispatch Using a Revised Linear Programming Approach,” Electric Power System Research, vol. 15, pp. 121-134, 1988.

[6] N. Grudinin, "Reactive Power Optimization Using Successive Quadratic Programming Method," IEEE Trans. on Power Systems, vol. 13, pp. 1219-1225, 1998.

[7] M. A. Abido, "Optimal Power Flow Using Particle Swarm Optimization," Electrical Power and Energy Systems, vol. 24, pp. 563-571, 2002.

[8] A. Abou El Ela, M. A. Abido, S. R. Spea, "Differential Evolution Algorithm for Optimal Reactive Power Dispatch,” Electric Power Systems Research, vol. 81, pp. 458-464, 2011.

[9] V. Miranda, N. Fonseca, "EPSO-Evolutionary Particle Swarm Optimization, A New Algorithm with Applications in Power Systems," in Proc. of Transmission and Distribution conf., vol. 2, pp. 745-750,2002.

[10] C.A. Canizares, A.C.Z.de Souza and V.H. Quintana, "Comparison of performance indices for detection of proximity to voltage collapse," vol. 11. no.3, pp.1441-1450, Aug 1996.

[11] E. Rashedi, H. Nezamabadi-pour, S. Saryazdi, "GSA: A gravitational search algorithm," Information Sciences, vol. 179, pp. 2232-2248, 2009.

[12] X. Lai and M. Zhang, "An efficient ensemble of GA and PSO for real function optimization," in 2nd IEEE International Conference on Computer Science and Information Technology, 2009, pp. 651-655.

[13] A. A. A. Esmin, G. Lambert-Torres, and G. B. Alvarenga, "Hybrid Evolutionary Algorithm Based on PSO and GA mutation," in proceeding of the Sixth International Conference on Hybrid Intelligent Systems (HIS 06), 2007, p. 57.

[14] L. Li, B. Xue, B. Niu, L. Tan, and J. Wang, "A Novel PSO-DE-Based Hybrid Algorithm for Global Optimization," in Lecture Notes in Computer Science.: Springer Berlin / Heidelberg, 2008, pp. 785-793.

[15] N. Holden and AA Freitas, "A Hybrid PSO/ACO Algorithm for Discovering Classification Rules in Data Mining," Journal of Artificial Evolution and Applications (JAEA), 2008.

[16] S. Durairaj, P. S. Kannan, D. Devaraj, "Application of Genetic Algorithm to Optimal Reactive Power Dispatch Including Voltage Stability Constraint," Journal of Energy \& Environment, vol. 4, pp. 63-73, 2005.

[17] J. Kennedy and RC. Eberhart, "Particle swarm optimization," in Proceedings of IEEE international conference on neural networks, vol. 4, 1995, pp. 1942-1948.

[18] Y. Shi and R.C. Eberhart, "A modified Particle Swarm Optimiser," inIEEE International Conference on Evolutionary Computation,Anchorage, Alaska, 1998.

[19] E. Rashedi, S. Nezamabadi, and S. Saryazdi, "GSA: A Gravitational Search Algorithm,"Information Sciences, vol. 179, no. 13, pp. 2232-2248, 2009.

[20] Wu Q H, Ma J T. "Power system optimal reactive power dispatch using evolutionary programming", IEEE Transactions on power systems 1995; 10(3): 1243-1248.

[21] S.Durairaj, D.Devaraj, P.S.Kannan "Genetic algorithm applications to optimal reactive power dispatch with voltage stability enhancement”, IE(I) Journal-EL Vol 87,September 2006.

[22] D.Devaraj, "Improved genetic algorithm for multi - objective reactive power dispatch problem", European Transactions on electrical power 2007; 17: 569-581.

[23] P. Aruna Jeyanthy and Dr. D. Devaraj "Optimal Reactive Power Dispatch for Voltage Stability Enhancement Using Real Coded Genetic Algorithm", International Journal of Computer and Electrical Engineering, Vol. 2, No. 4, August 2010 1793-8163.

*Corresponding author.

E-mail address: gklenin@ gmail.com 